REVIEW

This paper is dedicated to the $70^{\text {th }}$ anniversary of the founding of Physiologia Bohemoslovaca (currently Physiological Research)

\title{
Parental Overnutrition by Carbohydrates in Developmental Origins of Metabolic Syndrome
}

\author{
Ondřej ŠEDA ${ }^{1}$ \\ ${ }^{1}$ Institute of Biology and Medical Genetics, the First Faculty of Medicine, Charles University and \\ the General University Hospital, Prague, Czech Republic
}

Received September 20, 2021

Accepted November 5, 2021

\begin{abstract}
Summary
Metabolic syndrome is a prevalent disease resulting from an interplay of genomic component and the exposome. Parental diet has been shown to affect offspring metabolic health via multiple epigenetic mechanisms. Excess carbohydrate intake is one of the driving forces of the obesity and metabolic syndrome pandemics. This review summarizes the evidence for the effects of maternal carbohydrate (fructose, sucrose, glucose) overnutrition on the modulation of metabolic syndrome components in the offspring. Despite substantial discrepancies in experimental design, common effects of maternal carbohydrate overnutrition include increased body weight and hepatic lipid content of the "programmed" offspring. However, the administration of sucrose to several rat models leads to apparently favorable metabolic outcomes. Moreover, there is evidence for the role of genomic background in modulating the metabolic programming effect in the form of nutri-epigenomic interaction. Comprehensive, robust studies are needed to resolve the temporal, sex-specific, genetic, epigenetic and nutritional aspects of parental overnutrition in the intergenerational and transgenerational pathogenesis of metabolic syndrome.
\end{abstract}

\section{Key words}

Developmental origins of human adult diseases - Metabolic Syndrome • Sucrose • Fructose • Nutrigenetics

\section{Corresponding author}

Ondřej Šeda, Institute of Biology and Medical Genetics, First Faculty of Medicine, Charles University, Albertov 4, 12800 Prague 2, Czech Republic. E-mail: oseda@lf1.cuni.cz

\section{Metabolic syndrome}

Many epidemiological and clinical studies show consistent correlations between anthropometric parameters (obesity, visceral storage of body fat), metabolic markers (increased triacylglycerol concentration, hyperinsulinemia, glucose intolerance, low HDL cholesterol levels), and hemodynamic dysregulations (Kesaniemi et al. 1992). Finding that non-obese hypertensive individuals show more marked insulin resistance compared to non-obese normotensive subjects led Reaven (1988) to the introduction of the term "metabolic syndrome X." According to its original definition, the main components of metabolic syndrome (MetS) were: glucose intolerance, resistance to insulin-stimulated glucose uptake, hyperinsulinemia, hypertriglyceridemia, and hypertension. Over time, other conditions have been proposed as part of the metabolic syndrome including obesity, microalbuminuria (Palaniappan et al. 2003), hyperuricemia (Yanai et al. 2021), coagulation disorders (Meigs 2000) or increase in serum gammaglutamyl transferase (Godsland et al. 2005). So far, a number of definitions of metabolic syndrome have been proposed by different professional organizations. Among the most commonly used are the National Cholesterol Education Program's definitions Adult Treatment Panel III report (Grundy et al. 2004), WHO definition (Alberti and Zimmet 1998), and the worldwide consensus definition of International Diabetes Federation (Alberti et al. 2006). The latter definition introduces geoethnically specific criteria for assessing individual thresholds for the components of metabolic syndrome. Despite some differences between 
these definitions, resulting from the emphasis on either insulin resistance or obesity, there exists a considerable overlap between those diagnosed with metabolic syndrome by any of the criteria.

In this context, the debate whether metabolic syndrome per se constitutes a separate nosological unit and if its diagnosis somehow contributes to therapeutic decision-making compared to the consideration of individual findings has been long underway (Kahn et al. 2005). There is a considerable effort to transform the binary MetS into a continuous indicator using different MetS scores that should allow finer resolution for assessing MetS-conveyed risks, particularly that of type 2 diabetes and cardiovascular disease (DeBoer and Gurka, 2017). The prevalence of MetS is on the rise worldwide, affecting over $25 \%$ of the general population in westernized countries but also reaching "double-digit" percentages in developing regions, including sub-Saharan Africa (Jaspers Faijer-Westerink et al. 2020), Asia, or South America (Saklayen, 2018). In terms of its pathogenesis, metabolic syndrome is a cluster of multifactorial traits as each of its components has both substantial heritable component, which interacts with forces of the exposome, i.e., the totality of environmental exposures (Vermeulen et al. 2020). The dynamic nature of the gene-environmental architecture of MetS and its features was described both in humans and experimental model systems (Hamet et al. 2005, Seda et al. 2005).

\section{Complexity (dimensionality) reduction approaches in metabolic syndrome}

The detailed dissection of the intertwined network of genes and environmental factors (i.e., the ecogenomic architecture) of most complex conditions, including MetS, is complicated in general human populations for apparent reasons. Therefore, several approaches aimed at reducing the inherent complexity are being employed to elucidate the underlying mechanisms. For instance, genetically heterogeneous, complex metabolic and cardiovascular traits may need to be subdivided into clinically and genetically distinct subsets (Zak et al. 2014) to analyze their genomic architecture successfully. Another level of reduction involves the use of genetically designed experimental models. The use of mice and rats as experimental models has several advantages over many other possible complexity reduction approaches because of the physiological, anatomical, and genomic similarities between rodents and humans and the wealth of scientific data already available in these species (Aitman et al. 2016). Inbred animal strains are often used, representing genetically identical animals within one strain and gender, overcoming thus the genetic heterogeneity present in human cohorts (Sedova et al. 2000). Targeted manipulation of the rodent genome allows assessing the importance of particular genomic regions or gene variants in the pathogenesis of metabolic syndrome (Shamansurova et al. 2016, Sedova et al. 2021, Seda et al. 2002). Additional advantages include selective mating, relatively short generation time, and the possibility of standardization and specific manipulation of environmental conditions (e.g., diet, temperature, day/light regimen). Although the experimental findings are not directly transferable to the human situation and need to be validated (Seda et al. 2017), significant insights have been obtained through integrative and comparative genomics (Aitman et al. 2016). While rodent models represent the most common choice of experimental models for developmental origins of health and disease (DOHAD), other animal models systems are used to address specific questions based on physiological similarities of development (e.g., the sheep and non-human primates as models for developmental origins of polycystic ovary syndrome, Dumesic et al. 2020).

\section{Developmental origins of adult disease and metabolic syndrome.}

The "Thrifty Phenotype Hypothesis" (Hales and Barker 1992) perceives MetS in adults with a history of fetal malnutrition due to the predictive-adaptive strategies adopted by the fetus to maximize its chances of postnatal survival. Several other factors were identified as potential epigenetic modulators of cardiovascular disease, including maternal stress, maternal and early offspring nutrition, maternal smoking and alcohol abuse, litter size in rodent studies, etc. Despite considerable evidence for the developmental plasticity involvement in adult disease (Fleming et al. 2018), our understanding of the mechanisms underlying this process is still incomplete; an authoritative account of the current knowledge on DOHAD in metabolic disease was recently published (Hoffman et al. 2021). While the original DOHAD studies focused on malnutrition and placental-induced intrauterine growth restriction leading to catch-up growth and development of MetS features in adulthood in the affected offspring, maternal overnutrition seems to pose 
detrimental effects in this regard as well, suggesting a "U-shaped" pattern of risk. It was recently shown in a meta-analysis of 79 studies that pre-conception maternal obesity increases the odds of child obesity by $264 \%$ (Heslehurst et al. 2019). Maternal obesity is associated with a substantially higher risk of gestational diabetes mellitus, which in turn is associated with childhood increased adiposity (Lowe et al. 2019), impaired glucose tolerance, and high blood pressure (Tam et al. 2017). Furthermore, at least some of the effects of maternal obesity on offspring's metabolic dysfunction seem to be sex-specific (Dearden et al. 2018, Nicholas et al. 2020), concurring with the described sexspecific genome-environmental architecture of MetS and its components (Seda et al. 2008, Ohkuma et al. 2020).

\section{Epigenetic and genetic aspects developmental origins of adult disease}

The current understanding of the mechanisms, how the imbalanced prenatal environment triggers lasting changes in the exposed offspring involves modulation of gene expression by epigenetic cues (DNA methylation, histone acetylation, chromatin remodeling, small RNAs, particularly microRNAs and tRNA-derived fragments), and a detailed account on the molecular level and the current understanding of their trans- and intergenerational inheritance is beyond the scope of this review (for details, see the recent accounts of the topic (Hoffman et al. 2021, Galan et al. 2020)). Interestingly, there is a tighter interaction between epigenomic and genomic components in the pathogenetic process as some of the outcomes of epigenomic modulations depend on the genomic constitution (Pausova et al. 2003, Buresova et al. 2006, Skolnikova et al. 2020b). If proven true, the thrifty gene $\mathrm{x}$ thrifty phenotype (nature $\mathrm{x}$ nurture) dichotomy may eventually dissolve as these seemingly incompatible concepts can be integrated within a unifying gene-by-environmental network (Li et al. 2019). Utilizing many inbred, genetically distinct experimental rat strains may allow modeling, in a limited yet supervised fashion, the genetic diversity within the DOHAD. At the same time, this approach allows studying the epigenome modifications within genetically identical animals, i.e., in standardized genomic and environmental setup. In humans, the recent attempts to gauge the effect of genetic factors in DOHAD, e.g., using the Mendelian randomization approach (Moen et al. 2020), have been complicated by the inherent complexity mentioned earlier and by the methodological issues (D'Urso et al. 2021).

\section{Microbiome importance in developmental origins of adult disease}

Maternal microbiota contribute significantly to the initial microbial colonization of the neonate, with both short- and long-term impact (Calatayud et al. 2019). While there is ample evidence for nutritional modulation of microbiota (reviewed by Moszak et al. 2020), a detailed assessment of associations between maternal and offspring microbiome in connection to specific dietary regimens during pregnancy is missing. Most DOHAD studies do not involve metagenomic analyses assessing the microbial diversity aspects of early-life perturbations. The associations between the early gut microbiome and DOHAD were recently comprehensively reviewed (Sarkar et al. 2021).

\section{Paternal overnutrition}

While the potential effects of paternal environmental exposures and lifestyle in terms of programming the metabolic dysfunction of offspring have received less attention so far, an increasing body of evidence led to the establishment of the Paternal Origins of Health and Disease paradigm (Soubry, 2018). Sharp and Lawlor (2019) recently identified 47 studies showing a link between paternal adiposity (including BMI and high-fat diet-induced obesity) and obesity- or type 2 diabetes-related offspring outcomes, including birth weight, body fat, BMI, and obesity-related gene expression. However, the authors concluded that despite the accumulating data, more targeted research is needed to provide more robust causal evidence. Bodden et al. (2020) recently summarized the existing literature on diet-induced modification of sperm epigenome and its programming effect on offspring's metabolism, including the possible responsible mechanisms. These include dietinduced alterations in seminal plasma, qualitative and quantitative changes in sperm microRNAs, transfer RNAderived small RNAs, long non-coding RNAs, oxidativestress-induced sperm DNA damage or reduced sperm mitochondrial function. All the so far performed studies of paternal overnutrition involved high-fat or mixed highfat, high-sucrose diets, i.e., none involved diets enriched by carbohydrates only. So, the following section is focused only on maternal carbohydrate overnutrition.

\section{Maternal carbohydrate overnutrition}

The recent rise of increased carbohydrate intake 
is often related to the utilization of sucrose and high fructose corn syrup (HFCS) as sweeteners in soft drinks and processed foods (Sloboda et al. 2014). According to the available data, the use of HFCS increased over last several decades by over $1000 \%$ in spite of its clear connection to the pathogenesis of MetS and related metabolic complications (Hannou et al. 2018). Moreover, it was shown recently that feeding fructose drives an increase in the surface area of the gut that is associated with enhanced absorption of dietary nutrients and weight gain (Taylor et al. 2021). As evident from Tables 1-3, the studies on maternal carbohydrate overnutrition differ in many aspects making their comparison complicated. First, they include several distinct approaches in terms of overnutrition timing: from the most prolonged periods encompassing month prior conception till weaning of offspring to selected critical windows (Kunes et al. 2015) only, most commonly gestation or lactation. Second, both

Table 1. Maternal fructose overnutrition studies.

\begin{tabular}{|c|c|c|c|c|c|c|c|}
\hline Organism & Strain & $\begin{array}{c}\text { Maternal fructose } \\
\text { overnutrition }\end{array}$ & $\begin{array}{l}\text { Overnutrition } \\
\text { timing }\end{array}$ & $\begin{array}{l}\text { Offspring } \\
\text { age }\end{array}$ & $\begin{array}{c}\text { MetS-related offspring } \\
\text { effect }\end{array}$ & $\begin{array}{c}\text { Affected } \\
\text { generation }\end{array}$ & Reference \\
\hline \multirow[t]{2}{*}{ Mouse } & \multirow[t]{2}{*}{$\mathrm{C} 57 \mathrm{BL} / 6$} & \multirow[t]{2}{*}{ Liquid $20 \% \mathrm{w} / \mathrm{v}$} & \multirow[t]{2}{*}{$\begin{array}{l}\text { Gestation } \rightarrow \\
\text { Weaning }\end{array}$} & P210 & $\begin{array}{c}\mathrm{M}, \mathrm{F}: \mathrm{BW} \uparrow, \mathrm{Liv} \text { TG } \uparrow, \\
\mathrm{AST} \uparrow, \mathrm{TG} \uparrow, \mathrm{TC} \uparrow, \text { Glu } \uparrow, \\
\mathrm{SBP} \uparrow\end{array}$ & $\mathrm{F} 1$ & $\begin{array}{l}\text { (Koo et al. } \\
\text { 2021) }\end{array}$ \\
\hline & & & & $\mathrm{P} 240$ & $\begin{array}{l}\text { activation of renin- } \\
\text { angiotensin-aldosterone } \\
\text { system }\end{array}$ & F1-F4 & $\begin{array}{l}\text { (Seong et al. } \\
\text { 2019) }\end{array}$ \\
\hline \multirow[t]{10}{*}{ Rat } & \multirow[t]{8}{*}{ SD } & \multirow[t]{3}{*}{ Liquid $10 \% \mathrm{w} / \mathrm{v}$} & \multirow[t]{3}{*}{ Gestation } & $\mathrm{P} 0$ & TG $\downarrow$, Liv TG $\uparrow$ & \multirow[t]{10}{*}{$\mathrm{F} 1$} & $\begin{array}{c}\text { (Rodriguez et } \\
\text { al. 2013) }\end{array}$ \\
\hline & & & & P90 & $\begin{array}{c}\text { M: Lep } \uparrow, \text { Adiponectin } \uparrow, \\
\text { FPI } \uparrow ; \text { F: FPI } \downarrow\end{array}$ & & $\begin{array}{c}\text { (Rodriguez et } \\
\text { al. 2015) }\end{array}$ \\
\hline & & & & P261 & M: HDL-C $\uparrow ; \mathrm{F}: \mathrm{TC} \downarrow$ & & $\begin{array}{l}\text { (Rodrigo et } \\
\text { al. 2018) }\end{array}$ \\
\hline & & \multirow[t]{2}{*}{ Liquid $20 \% \mathrm{w} / \mathrm{v}$} & \multirow[t]{2}{*}{$\begin{array}{l}12 \text { weeks } \\
\text { pre-conception } \\
\rightarrow \text { weaning }\end{array}$} & \multirow[t]{2}{*}{$\mathrm{P} 21$} & $\begin{array}{l}\text { FPG } \uparrow, \text { FINS } \uparrow, \text { HOMA-IR } \\
\uparrow, \text { TG } \uparrow, \text { NEFA } \uparrow, \text { LivTG } \uparrow\end{array}$ & & $\begin{array}{c}\text { (Yuruk and } \\
\text { Nergiz-Unal, } \\
\text { 2017) }\end{array}$ \\
\hline & & & & & Body fat $\uparrow$, Leptin $\downarrow$ & & $\begin{array}{l}\text { (Kisioglu and } \\
\text { Nergiz-Unal, } \\
\text { 2020) }\end{array}$ \\
\hline & & \multirow[t]{2}{*}{ Solid $(50 \% \mathrm{w} / \mathrm{w})$} & $\begin{array}{l}2 \text { weeks } \\
\text { pre-conception } \\
\rightarrow \text { birth }\end{array}$ & P0, P22 & $\mathrm{M} / \mathrm{F}: \mathrm{Glu} \uparrow$ at $\mathrm{P} 0$ & & $\begin{array}{l}\text { (Jen et al. } \\
\text { 1991) }\end{array}$ \\
\hline & & & \multirow[t]{2}{*}{$\begin{array}{l}\text { Gestation } \rightarrow \\
\text { Weaning }\end{array}$} & P87 & $\begin{array}{c}\text { M,F: BW } \uparrow \text {, Adiposity } \uparrow \text {, } \\
\text { TG } \uparrow, \text { TC } \uparrow, \text { FPG } \uparrow, \text { FINS } \\
\uparrow, \text { Lep } \uparrow, \text { Adiponectin } \downarrow\end{array}$ & & $\begin{array}{l}\text { (Tobar- } \\
\text { Bernal et al. } \\
\text { 2021) }\end{array}$ \\
\hline & & Solid $(60 \% \mathrm{w} / \mathrm{w})$ & & P84 & $\mathrm{M}: \mathrm{SBP} \uparrow$ & & $\begin{array}{l}\text { (Tain et al. } \\
\text { 2015), (Tain } \\
\text { et al. 2018) }\end{array}$ \\
\hline & \multirow[t]{2}{*}{ Wistar } & $\begin{array}{c}\text { Liquid, } \\
20 \text { cal\% fructose }\end{array}$ & \multirow[t]{2}{*}{$\begin{array}{l}\text { Gestation } \rightarrow \\
\text { P10 }\end{array}$} & \multirow[t]{2}{*}{$\mathrm{P} 10$} & $\begin{array}{c}\text { M: BW } \uparrow, \text { Glu } \downarrow \text {, Ins } \downarrow \\
\text { F: BW } \uparrow, \text { Ins } \downarrow \text {, Lep } \uparrow\end{array}$ & & $\begin{array}{c}\text { (Vickers et al. } \\
\text { 2011) }\end{array}$ \\
\hline & & $\begin{array}{c}\text { Crystalline, } \\
20 \text { cal\% fructose }\end{array}$ & & & $\begin{array}{c}\text { M: ALP } \uparrow ; \text { M,F: TP } \downarrow ; \\
\text { M,F: LivTG } \uparrow\end{array}$ & & $\begin{array}{l}\text { (Clayton et } \\
\text { al. 2015) }\end{array}$ \\
\hline
\end{tabular}

SD: Sprague Dawley rat, P: postnatal day, M: male, F: female, BW: bodyweight, LivTG: Liver triacyglycerols, AST: Aspartate aminotransferase, TG: triacylglycerols, TC: total cholesterol, Glu: glycemia, SBP: systolic blood pressure, Lep: leptin, FPI: fasting plasma insulin, HDL-C: high-density lipoprotein cholesterol, FPG: fasting plasma glucose, HOMA-IR: Homeostatic Model Assessment for Insulin Resistance, NEFA: non-esterified free acids, Ins: insulin, ALP: alkaline phosphatase, TP: total protein, F1-F4: filial generation 1-4. 
Table 2. Maternal sucrose overnutrition studies.

\begin{tabular}{|c|c|c|c|c|c|c|c|}
\hline Organism & Strain & $\begin{array}{c}\text { Maternal } \\
\text { sucrose } \\
\text { overnutrition } \\
\end{array}$ & $\begin{array}{l}\text { Overnutrition } \\
\text { timing }\end{array}$ & $\begin{array}{l}\text { Offspring } \\
\text { age }\end{array}$ & $\begin{array}{l}\text { MetS-related offspring } \\
\text { effect }\end{array}$ & $\begin{array}{l}\text { Gene- } \\
\text { ration }\end{array}$ & Reference \\
\hline \multirow[t]{17}{*}{ Rat } & \multirow[t]{3}{*}{ BHE } & \multirow{3}{*}{$\begin{array}{l}\text { Solid diet with } \\
65 \% \text { sucrose }\end{array}$} & Gestation & \multirow[t]{3}{*}{ P142 } & M: TG $\downarrow$, Ins $\downarrow$ & \multirow[t]{6}{*}{$\mathrm{F} 1$} & (Berdanier, \\
\hline & & & Lactation & & M: TG $\downarrow, \mathrm{BW}$ gain $\downarrow$ & & \\
\hline & & & Gestation $\rightarrow$ Weaning & & M: TG $\downarrow$, Liv lip $\uparrow$, Ins $\downarrow$ & & \\
\hline & $\mathrm{SD}$ & $\begin{array}{l}\text { Solid diet with } \\
50 \% \text { sucrose }\end{array}$ & $\begin{array}{l}2 \text { weeks } \\
\text { pre-conception } \\
\rightarrow \text { birth }\end{array}$ & P0, P22 & $\mathrm{M} / \mathrm{F}: \mathrm{Glu} \uparrow$ at $\mathrm{P} 0$ & & $\begin{array}{l}\text { (Jen et al. } \\
1991)\end{array}$ \\
\hline & PD & Solid, $70 \mathrm{cal} \%$ & Gestation $\rightarrow$ Weaning & $\begin{array}{l}\text { P0, P21, } \\
\text { P140 }\end{array}$ & $\begin{array}{c}\text { M(P0): Glu } \downarrow ; \\
\text { M(P21): BW } \uparrow, \\
\text { Adiponectin } \uparrow ; \\
\text { M (P140): Adiposity } \uparrow, \\
\text { LDL-C } \uparrow, \text { Adiponectin } \uparrow\end{array}$ & & $\begin{array}{c}\text { (Sedova et al. } \\
\text { 2007) }\end{array}$ \\
\hline & \multirow[t]{2}{*}{$\begin{array}{c}\text { SHR, } \\
\text { SHR.PD }\end{array}$} & & & \multirow[t]{2}{*}{ P180 } & $\mathrm{M}: \mathrm{BAT} \uparrow, \mathrm{LDL}-\mathrm{C} \downarrow$ & & $\begin{array}{l}\text { (Skolnikova } \\
\text { et al. 2020a) }\end{array}$ \\
\hline & & & & & $\begin{array}{c}\mathrm{M}_{\mathrm{SHR}}: \mathrm{BAT} \uparrow, \mathrm{FPG} \downarrow, \\
\mathrm{HDL}-\mathrm{C} \uparrow \\
\mathrm{M}_{\mathrm{SHR} . \mathrm{PD}}: \mathrm{BAT} \uparrow, \mathrm{FINS} \uparrow, \\
\mathrm{HDL}-\mathrm{C} \uparrow\end{array}$ & $\mathrm{F} 2$ & $\begin{array}{l}\text { (Skolnikova } \\
\text { et al. 2020c) }\end{array}$ \\
\hline & \multirow[t]{5}{*}{ Wistar } & \multirow[t]{5}{*}{$\begin{array}{l}\text { Liquid } \\
10 \% \mathrm{w} / \mathrm{v}\end{array}$} & Gestation $\rightarrow$ Weaning & P21, P84 & $\begin{array}{c}\text { Hepatic Srebplc } \\
\text { expression } \uparrow\end{array}$ & \multirow[t]{10}{*}{$\mathrm{F} 1$} & $\begin{array}{l}\text { (Kaur et al. } \\
\text { 2018) }\end{array}$ \\
\hline & & & $\begin{array}{l}4 \text { weeks } \\
\text { pre-conception } \\
\rightarrow \text { birth }\end{array}$ & P60-P90 & M,F: no effect & & $\begin{array}{l}\text { (Kendig et al. } \\
\text { 2015) }\end{array}$ \\
\hline & & & $\begin{array}{l}4 \text { weeks } \\
\text { pre-conception } \\
\rightarrow \text { weaning }\end{array}$ & \multirow[t]{3}{*}{ P21, P84 } & $\begin{array}{c}\text { F(P21):Adiposity } \uparrow \text {; Glu } \downarrow ; \\
\text { M(P21): Adiposity } \uparrow ; \\
\text { Glu } \downarrow \text {; TG } \downarrow ; \\
\text { M(P84): Adiposity } \downarrow ; \\
\text { FFA } \uparrow ; \\
\text { F(P84): Adiposity } \downarrow\end{array}$ & & \multirow[t]{3}{*}{$\begin{array}{l}\text { (Toop et al. } \\
\text { 2017) }\end{array}$} \\
\hline & & & $\begin{array}{l}4 \text { weeks } \\
\text { pre-conception } \\
\rightarrow \text { birth }\end{array}$ & & $\begin{array}{c}\text { M(P21): Glu } \downarrow, \text { TG } \downarrow \text {, } \\
\text { HDL-C } \uparrow ; \\
\text { F(P21): Glu } \downarrow, \text { HDLC } \uparrow \\
\text { M(P84): Adiposity } \downarrow ; \\
\text { FFA } \uparrow ; \\
\text { F(P84): Adiposity } \downarrow\end{array}$ & & \\
\hline & & & $\begin{array}{c}4 \text { weeks } \\
\text { pre-conception }+ \\
\text { lactation }\end{array}$ & & $\begin{array}{c}\text { M(P21): TG } \uparrow, \text { HDL-C } \downarrow ; \\
\text { F(P21): Glu } \downarrow ; \\
\text { M(P84): FFA } \uparrow ; \\
\text { F(P84): Adiposity } \downarrow\end{array}$ & & \\
\hline & \multirow[t]{3}{*}{ N.A. } & $\begin{array}{l}\text { Liquid } \\
10 \% \mathrm{w} / \mathrm{v}\end{array}$ & \multirow[t]{3}{*}{$N . A$. } & \multirow[t]{3}{*}{ N.A. } & $\mathrm{BW} \uparrow$ & & \multirow[t]{3}{*}{$\begin{array}{c}\text { (Ozkan et al. } \\
\text { 2019) }\end{array}$} \\
\hline & & $20 \% \mathrm{w} / \mathrm{v}$ & & & $\mathrm{BW} \uparrow$, Glu $\uparrow$, Ins $\uparrow$ & & \\
\hline & & $30 \% \mathrm{w} / \mathrm{v}$ & & & $\mathrm{BW} \uparrow$, Glu $\uparrow$, Ins $\uparrow$ & & \\
\hline & \multirow[t]{2}{*}{ SD } & \multirow[t]{2}{*}{$\begin{array}{l}\text { Liquid } \\
20 \% \mathrm{w} / \mathrm{v}\end{array}$} & \multirow[t]{2}{*}{$\begin{array}{l}12 \text { weeks } \\
\text { pre-conception } \\
\rightarrow \text { weaning }\end{array}$} & \multirow[t]{2}{*}{$\mathrm{P} 21$} & $\mathrm{TG} \uparrow, \mathrm{NEFA} \uparrow, \mathrm{Liv} \mathrm{TG} \uparrow$ & & $\begin{array}{l}\text { (Yuruk and } \\
\text { Nergiz-Unal, } \\
\text { 2017) }\end{array}$ \\
\hline & & & & & Body fat $\uparrow$ & & $\begin{array}{l}\text { (Kisioglu and } \\
\text { Nergiz-Unal, } \\
\text { 2020) }\end{array}$ \\
\hline
\end{tabular}


Table 2. Maternal sucrose overnutrition studies. (Continued)

\begin{tabular}{|c|c|c|c|c|c|c|c|}
\hline Organism & Strain & $\begin{array}{c}\text { Maternal } \\
\text { sucrose } \\
\text { overnutrition } \\
\end{array}$ & $\begin{array}{c}\text { Overnutrition } \\
\text { timing }\end{array}$ & $\begin{array}{l}\text { Offspring } \\
\text { age }\end{array}$ & $\begin{array}{c}\text { MetS-related offspring } \\
\text { effect }\end{array}$ & $\begin{array}{l}\text { Gene- } \\
\text { ration }\end{array}$ & Reference \\
\hline & \multirow[t]{7}{*}{ SD } & \multirow[t]{7}{*}{$\begin{array}{l}\text { Liquid } \\
20 \% \mathrm{w} / \mathrm{v}\end{array}$} & \multirow[t]{7}{*}{ Gestation } & $\mathrm{P} 14, \mathrm{P} 180$ & $\mathrm{BW} \uparrow$ & & $\begin{array}{c}\text { (Bocarsly et } \\
\text { al. 2012) }\end{array}$ \\
\hline & & & & P30, P60 & BW $\uparrow$, brain weight $\downarrow$ & & $\begin{array}{l}\text { (Kuang et } \\
\text { al. 2014) }\end{array}$ \\
\hline & & & & P150 & $\mathrm{M}: \mathrm{BW} \uparrow, \mathrm{FPG} \uparrow$ & & $\begin{array}{c}\text { (Gu et al. } \\
\text { 2017) }\end{array}$ \\
\hline & & & & P150 & $\begin{array}{c}\text { M: Ang II-mediated } \\
\text { oressir response and } \\
\text { vessel tone } \uparrow\end{array}$ & & $\begin{array}{l}\text { (Wu et al. } \\
\text { 2014) }\end{array}$ \\
\hline & & & & P180 & M: GT $\downarrow$, LI area $\uparrow$ & & $\begin{array}{l}\text { (Zhang et } \\
\text { al. 2018) }\end{array}$ \\
\hline & & & & P540 & $\begin{array}{c}\text { M: BW } \uparrow, \text { FINS } \uparrow, \\
\text { HOMA-IR } \uparrow, \\
\text { oxidative stress } \uparrow\end{array}$ & & $\begin{array}{l}\text { (He et al. } \\
\text { 2017) }\end{array}$ \\
\hline & & & & P660 & $\mathrm{SBP} \uparrow, \mathrm{DBP} \uparrow, \mathrm{MAP} \uparrow$ & & $\begin{array}{c}\text { (Wu et al. } \\
2016)\end{array}$ \\
\hline
\end{tabular}

BHE: Bureau of Home Economics rat strain, SD: Sprague Dawley rat, PD: polydactylous rat strain, SHR: spontaneously hypertensive strain, P: postnatal day, N.A.: not available, M: male, F: female, TG: triacylglycerols, Ins: insulin, BW: bodyweight, Liv lip: Liver lipids, Glu: glycemia, LDL-C: low-density lipoprotein cholesterol, BAT: brown adipose tissue, FPG: fasting plasma glucose, HDL-C: high-density lipoprotein cholesterol, FPI: fasting plasma insulin, FFA: free fatty acids, NEFA: non-esterified free acids, Liv TG: liver triacylglycerols, AngII: angiotensin II, GT: glucose tolerance, LI: Langerhans islets, HOMA-IR: Homeostatic Model Assessment for Insulin Resistance, SBP: systolic blood pressure, DBP: diastolic blood pressure, MAP: mean arterial blood pressure. TP: total protein, F1-F2: filial generation 1-2.

ways of administering excess carbohydrates, i.e., in liquid and solid form, have been used in the annotated metabolic programming studies (Tables 1-3). There is currently no consensus on the optimal approach regarding its translational relevance to human conditions, as recently discussed by Eng and Estall (2021). Even though studies directly comparing the liquid and solid administration of fructose or sucrose in the same model under the same protocol are not available, it seems that the effects concerning the manifestation of MetS attributes in programmed offspring are similar. Third, different rodent strains are used, though Sprague-Dawley and Wistar rat strains seem to be the most common models for maternal carbohydrate overnutrition. As mentioned above, the genomic background upon which the nutri-epigenomic stimuli are acting can modify the ultimate programming effect. In almost all studies, only the intergenerational effects targeting directly F1 generation are studied. In a set of experiments, it has been shown that sucrose feeding of rat dams of two inbred strains affects two subsequent generations of male offspring (F1, F2) with similar outcomes, including the increase of brown fat adipose tissue depots and similar shifts in lipid profile (Skolnikova et al. 2020c, Skolnikova et al. 2020a). In the only truly transgenerational inheritance study, Seong et al. (2019) showed that administration of $20 \%$ fructose in drinking water induced multigenerational (down to F4) activation of the renin-angiotensin-aldosterone system as well as sodium transporters, leading to increased oxidative stress factors and inflammatory cytokines (Seong et al. 2019). Only two studies assessed, within a complex study design, specific overnutrition by administration of $10 \%$ glucose in the drinking water during the gestation of SD rats only (Table 3 ). The three-month-old male progeny showed increased adiponectin and lower fasting plasma insulin (Rodriguez et al. 2015), and the offspring of both sexes had decreased liver cholesterol (Rodrigo et al. 2018).

Despite the described discrepancies in experimental design, several common effects are observable. For instance, in the offspring of fructose and sucrose-fed mothers, increase of body weight is often present, particularly if the exposition encompassed both gestation and complete lactation periods. A possible mechanism recently suggested based on results from 
Table 3. Maternal glucose overnutrition studies.

\begin{tabular}{|c|c|c|c|c|c|c|c|}
\hline Organism & Strain & $\begin{array}{c}\text { Maternal } \\
\text { glucose } \\
\text { overnutrition }\end{array}$ & $\begin{array}{c}\text { Overnutrition } \\
\text { timing }\end{array}$ & $\begin{array}{c}\text { Offspring } \\
\text { age }\end{array}$ & $\begin{array}{c}\text { MetS-related } \\
\text { offspring effect }\end{array}$ & $\begin{array}{c}\text { Affected } \\
\text { generation }\end{array}$ & Reference \\
\hline Rat & SD & Liquid $10 \% \mathrm{w} / \mathrm{v}$ & Gestation & P90 & $\begin{array}{c}\text { M: Adiponectin } \uparrow, ~ \\
\text { F: FPI } \downarrow\end{array}$ & F1 & $\begin{array}{c}\text { (Rodriguez } \\
\text { et al. 2015) }\end{array}$ \\
\hline Rat & SD & Liquid $10 \% \mathrm{w} / \mathrm{v}$ & Gestation & P261 & M,F: Liv C $\downarrow$ & F1 & $\begin{array}{c}\text { (Rodrigo et } \\
\text { al. 2018) }\end{array}$ \\
\hline
\end{tabular}

SD: Sprague Dawley rat, P: postnatal day, M: male, F: female, FPI: fasting plasma insulin, Liv C: liver cholesterol, F1: filial generation 1.

EPOCH cohort involved early insulin hypersecretion as a major contributing process in the development of childhood obesity in children exposed to maternal overnutrition (Perng et al. 2021). This is corroborated by increased offspring insulinemia found in the same experiments (Table 1). Increased hepatic triacylglycerol content of the programmed offspring is the second most common finding in the fructose maternal overfeeding studies. It was shown that the fetal liver and hepatic mitochondrial function are particularly susceptible to dysregulated maternal fuel metabolism and increased hepatic lipid stores, disrupted mitochondria, and elevated oxidative stress ensue, eventually leading to nonalcoholic fatty liver disease, which is in turn tightly interconnected with MetS (Baker and Friedman, 2018). The administration of sucrose in the form of a solid diet or $10 \%$ solution to rat dams of several different rat models led, surprisingly, to apparently favorable metabolic changes, including a decrease in glycemia, triacylglycerols or LDL-cholesterol or increase of HDL-cholesterol. Glucose overfeeding solely during gestation formed part of only two studies (Table 3). Consistent with the different metabolic effects of postnatal administration of glucose vs. fructose (Softic et al. 2017), the offspring showed dissimilarities concerning the effects on circulating and tissue stores of lipids.

In conclusion, maternal carbohydrate overnutrition leads, in numerous rodent models and different overnutrition timing schemes, to substantial alterations of the metabolic profile of the offspring and often results in the manifestation of MetS or its individual components. Nevertheless, the effect of the metabolic programming by carbohydrates is clearly dependent on multiple factors, including the genomic background it acts upon, and in specific combinations, it may result even in metabolically favorable outcomes. Whether these observations represent the proposed predictive-adaptive responses due to mismatch of pre- and postnatal environments (Gluckman et al. 2019), or strain-specific, nutri-epigenomic interactions remains to be elucidated by conducting rigorous, full-factorial studies.

\section{Conflict of Interest}

There is no conflict of interest.

\section{Acknowledgements}

This work was supported by the Ministry of Health, Czech Republic - conceptual development of research organization MH CZ-DRO-VFN64165, General University Hospital in Prague, Czech Republic).

\section{References}

AITMAN T, DHILLON P, GEURTS AM: A RATional choice for translational research? Dis Model Mech 9: 10691072, 2016. https://doi.org/10.1242/dmm.027706

ALBERTI KG, ZIMMET P, SHAW J: Metabolic syndrome--a new world-wide definition. A consensus statement from the international diabetes federation. Diabet Med 23: 469-480, 2006. https://doi.org/10.1111/j.14645491.2006.01858.x

ALBERTI KG, ZIMMET PZ: Definition, diagnosis and classification of diabetes mellitus and its complications. Part 1: diagnosis and classification of diabetes mellitus provisional report of a WHO consultation. Diabet Med 15: 539-553, 1998. https://doi.org/10.1002/(SICI)1096-9136(199807)15:7<539::AID-DIA668>3.0.CO;2-S

BAKER PR, 2ND, FRIEDMAN JE: Mitochondrial role in the neonatal predisposition to developing nonalcoholic fatty liver disease. J Clin Invest 128: 3692-3703, 2018. https://doi.org/10.1172/JCI120846 
BERDANIER CD: Effect of maternal sucrose intake on the metabolic patterns of mature rat progeny. Am J Clin Nutr 28: 1416-1421, 1975. https://doi.org/10.1093/ajen/28.12.1416

BOCARSLY ME, BARSON JR, HAUCA JM, HOEBEL BG, LEIBOWITZ SF, AVENA NM: Effects of perinatal exposure to palatable diets on body weight and sensitivity to drugs of abuse in rats. Physiol Behav 107: 568-575, 2012. https://doi.org/10.1016/j.physbeh.2012.04.024

BODDEN C, HANNAN AJ, REICHELT AC: Diet-induced modification of the sperm epigenome programs metabolism and behavior. Trends Endocrinol Metab 31: 131-149, 2020. https://doi.org/10.1016/j.tem.2019.10.005

BURESOVA M, ZIDEK V, MUSILOVA A, SIMAKOVA M, FUCIKOVA A, BILA V, KREN V, KAZDOVA L, DI NICOLANTONIO R, PRAVENEC M: Genetic relationship between placental and fetal weights and markers of the metabolic syndrome in rat recombinant inbred strains. Physiol Genomics 26: 226-231, 2006. https://doi.org/10.1152/physiolgenomics.00056.2006

CALATAYUD M, KOREN O, COLLADO MC: Maternal microbiome and metabolic health program microbiome development and health of the offspring. Trends Endocrinol Metab 30: 735-744, 2019. https://doi.org/10.1016/j.tem.2019.07.021

CLAYTON ZE, VICKERS MH, BERNAL A, YAP C, SLOBODA DM: Early life exposure to fructose alters maternal, fetal and neonatal hepatic gene expression and leads to sex-dependent changes in lipid metabolism in rat offspring. PLoS One 10: e0141962, 2015. https://doi.org/10.1371/journal.pone.0141962

D'URSO S, WANG G, HWANG LD, MOEN GH, WARRINGTON NM, EVANS DM: A cautionary note on using Mendelian randomization to examine the Barker hypothesis and Developmental Origins of Health and Disease (DOHaD). J Dev Orig Health Dis 12: 688-693, 2021. https://doi.org/10.1017/S2040174420001105

DEARDEN L, BOURET SG, OZANNE SE: Sex and gender differences in developmental programming of metabolism. Mol Metab 15: 8-19, 2018. https://doi.org/10.1016/j.molmet.2018.04.007

DEBOER MD, GURKA MJ: Clinical utility of metabolic syndrome severity scores: considerations for practitioners. Diabetes Metab Syndr Obes 10: 65-72, 2017. https://doi.org/10.2147/DMSO.S101624

DUMESIC DA, HOYOS LR, CHAZENBALK GD, NAIK R, PADMANABHAN V, ABBOTT DH: Mechanisms of intergenerational transmission of polycystic ovary syndrome. Reproduction 159: R1-R13, 2020. https://doi.org/10.1530/REP-19-0197

ENG JM, ESTALL JL: Diet-induced models of non-alcoholic fatty liver disease: food for thought on sugar, fat, and cholesterol. Cells 10: 1805, 2021. https://doi.org/10.3390/cells10071805

FLEMING TP, WATKINS AJ, VELAZQUEZ MA, MATHERS JC, PRENTICE AM, STEPHENSON J, BARKER M, SAFFERY R, YAJNIK CS, ECKERT JJ, HANSON MA, FORRESTER T, GLUCKMAN PD, GODFREY KM: Origins of lifetime health around the time of conception: causes and consequences. Lancet 391: 1842-1852, 2018. https://doi.org/10.1016/S0140-6736(18)30312-X

GALAN C, KRYKBAEVA M, RANDO OJ: Early life lessons: The lasting effects of germline epigenetic information on organismal development. Mol Metab 38: 100924, 2020. https://doi.org/10.1016/j.molmet.2019.12.004

GLUCKMAN PD, HANSON MA, LOW FM: Evolutionary and developmental mismatches are consequences of adaptive developmental plasticity in humans and have implications for later disease risk. Philos Trans R Soc Lond B Biol Sci 374: 20180109, 2019. https://doi.org/10.1098/rstb.2018.0109

GODSLAND IF, CROOK D, PROUDLER AJ, STEVENSON JC: Hemostatic risk factors and insulin sensitivity, regional body fat distribution, and the metabolic syndrome. J Clin Endocrinol Metab 90: 190-197, 2005. https://doi.org/10.1210/jc.2004-1292

GRUNDY SM, CLEEMAN JI, MERZ CN, BREWER HB, JR., CLARK LT, HUNNINGHAKE DB, PASTERNAK RC, SMITH SC, JR., STONE NJ, NATIONAL HEART L, BLOOD I, AMERICAN COLLEGE OF CARDIOLOGY F, AMERICAN HEART A: Implications of recent clinical trials for the National Cholesterol Education Program Adult Treatment Panel III guidelines. Circulation 110: 227-239, 2004. https://doi.org/10.1161/01.CIR.0000133317.49796.0E

GU X, HE A, FAN X, SHI R, FENG X, BO L, JIANG L, LI N, WU J, YANG Y, GAO Q, XU Z: Regulation of cerebral arterial $\mathrm{BKCa}$ channels by angiotensin II signaling in adult offspring exposed to prenatal high sucrose diets. Biosci Rep 37: BSR20160624, 2017. https://doi.org/10.1042/BSR20160624 
HALES CN, BARKER DJ: Type 2 (non-insulin-dependent) diabetes mellitus: the thrifty phenotype hypothesis. Diabetologia 35: 595-601, 1992. https://doi.org/10.1007/BF00400248

HAMET P, MERLO E, SEDA O, BROECKEL U, TREMBLAY J, KALDUNSKI M, GAUDET D, BOUCHARD G, DESLAURIERS B, GAGNON F, ANTONIOL G, PAUSOVA Z, LABUDA M, JOMPHE M, GOSSARD F, TREMBLAY G, KIROVA R, TONELLATO P, ORLOV SN, PINTOS J, PLATKO J, HUDSON TJ, RIOUX JD, KOTCHEN TA, COWLEY AW, JR.: Quantitative founder-effect analysis of French Canadian families identifies specific loci contributing to metabolic phenotypes of hypertension. Am J Hum Genet 76: 815-832, 2005. https://doi.org/10.1086/430133

HANNOU SA, HASLAM DE, MCKEOWN NM, HERMAN MA: Fructose metabolism and metabolic disease. J Clin Invest 128: 545-555, 2018. https://doi.org/10.1172/JCI96702

HE A, ZHANG Y, YANG Y, LI L, FENG X, WEI B, ZHU D, LIU Y, WU L, ZHANG L, XU Z, SUN M: Prenatal high sucrose intake affected learning and memory of aged rat offspring with abnormal oxidative stress and NMDARs/Wnt signaling in the hippocampus. Brain Res 1669: 114-121, 2017. https://doi.org/10.1016/j.brainres.2017.05.022

HESLEHURST N, VIEIRA R, AKHTER Z, BAILEY H, SLACK E, NGONGALAH L, PEMU A, RANKIN J: The association between maternal body mass index and child obesity: A systematic review and meta-analysis. PLoS Med 16: e1002817, 2019. https://doi.org/10.1371/journal.pmed.1002817

HOFFMAN DJ, POWELL TL, BARRETT ES, HARDY DB: Developmental origins of metabolic diseases. Physiol Rev 101: 739-795, 2021. https://doi.org/10.1152/physrev.00002.2020

JASPERS FAIJER-WESTERINK H, KENGNE AP, MEEKS KAC, AGYEMANG C: Prevalence of metabolic syndrome in sub-Saharan Africa: A systematic review and meta-analysis. Nutr Metab Cardiovasc Dis 30: 547-565, 2020. https://doi.org/10.1016/j.numecd.2019.12.012

JEN KL, ROCHON C, ZHONG SB, WHITCOMB L: Fructose and sucrose feeding during pregnancy and lactation in rats changes maternal and pup fuel metabolism. J Nutr 121: 1999-2005, 1991. https://doi.org/10.1093/jn/121.12.1999

KAHN R, BUSE J, FERRANNINI E, STERN M: The Metabolic Syndrome: Time for a Critical Appraisal. Joint statement from the American Diabetes Association and the European Association for the Study of Diabetes 28: 2289-2304, 2005. https://doi.org/10.2337/diacare.28.9.2289

KAUR H, TOOP CR, MUHLHAUSLER BS, GENTILI S: The effect of maternal intake of sucrose or high-fructose corn syrup (HFCS)-55 during gestation and lactation on lipogenic gene expression in rat offspring at 3 and 12 weeks of age. J Dev Orig Health Dis 9: 481-486, 2018. https://doi.org/10.1017/S2040174418000260

KENDIG MD, EKAYANTI W, STEWART H, BOAKES RA, ROONEY K: Metabolic effects of access to sucrose drink in female rats and transmission of some effects to their offspring. PLoS One 10: e0131107, 2015. https://doi.org/10.1371/journal.pone.0131107

KESANIEMI YA, LILJA M, KERVINEN K, RANTALA A: Multiple metabolic syndrome: aspects of genetic epidemiology and molecular genetics. Ann Med 24: 461-464, 1992. https://doi.org/10.3109/07853899209166996

KISIOGLU B, NERGIZ-UNAL R: Potential effect of maternal dietary sucrose or fructose syrup on CD36, leptin, and ghrelin-mediated fetal programming of obesity. Nutr Neurosci 23: 210-220, 2020. https://doi.org/10.1080/1028415X.2018.1491151

KOO S, KIM M, CHO HM, KIM I: Maternal high-fructose intake during pregnancy and lactation induces metabolic syndrome in adult offspring. Nutr Res Pract 15: 160-172, 2021. https://doi.org/10.4162/nrp.2021.15.2.160

KUANG H, SUN M, LV J, LI J, WU C, CHEN N, BO L, WEI X, GU X, LIU Z, MAO C, XU Z: Hippocampal apoptosis involved in learning deficits in the offspring exposed to maternal high sucrose diets. J Nutr Biochem 25: 985-990, 2014. https://doi.org/10.1016/j.jnutbio.2014.04.012

KUNES J, VANECKOVA I, MIKULASKOVA B, BEHULIAK M, MALETINSKA L, ZICHA J: Epigenetics and a new look on metabolic syndrome. Physiol Res 64: 611-620, 2015. https://doi.org/10.33549/physiolres.933174

LI J, LI X, ZHANG S, SNYDER M: Gene-environment interaction in the era of precision medicine. Cell 177: 38-44, 2019. https://doi.org/10.1016/j.cell.2019.03.004 
LOWE WL, JR., LOWE LP, KUANG A, CATALANO PM, NODZENSKI M, TALBOT O, TAM WH, SACKS DA, MCCANCE D, LINDER B, LEBENTHAL Y, LAWRENCE JM, LASHLEY M, JOSEFSON JL, HAMILTON J, DEEROCHANAWONG C, CLAYTON P, BRICKMAN WJ, DYER AR, SCHOLTENS DM, METZGER BE, GROUP HF-USCR: Maternal glucose levels during pregnancy and childhood adiposity in the Hyperglycemia and Adverse Pregnancy Outcome Follow-up Study. Diabetologia 62: 598-610, 2019. https://doi.org/10.1007/s00125-018-4809-6

MEIGS JB: Invited commentary: insulin resistance syndrome? Syndrome X? Multiple metabolic syndrome? A syndrome at all? Factor analysis reveals patterns in the fabric of correlated metabolic risk factors. Am J Epidemiol 152: 908-911; discussion 912, 2000. https://doi.org/10.1093/aje/152.10.908

MOEN GH, BRUMPTON B, WILLER C, ASVOLD BO, BIRKELAND KI, WANG G, NEALE MC, FREATHY RM, SMITH GD, LAWLOR DA, KIRKPATRICK RM, WARRINGTON NM, EVANS DM: Mendelian randomization study of maternal influences on birthweight and future cardiometabolic risk in the HUNT cohort. Nat Commun 11: 5404, 2020. https://doi.org/10.1038/s41467-020-19257-z

NICHOLAS LM, NAGAO M, KUSINSKI LC, FERNANDEZ-TWINN DS, ELIASSON L, OZANNE SE: Exposure to maternal obesity programs sex differences in pancreatic islets of the offspring in mice. Diabetologia 63: 324-337, 2020. https://doi.org/10.1007/s00125-019-05037-y

OHKUMA T, PETERS SAE, JUN M, HARRAP S, COOPER M, HAMET P, POULTER N, CHALMERS J, WOODWARD M, GROUP AC: Sex-specific associations between cardiovascular risk factors and myocardial infarction in patients with type 2 diabetes: The ADVANCE-ON study. Diabetes Obes Metab 22: 1818-1826, 2020. https://doi.org/10.1111/dom.14103

OZKAN H, TOPSAKAL S, OZMEN O: Investigation of the diabetic effects of maternal high-glucose diet on rats. Biomed Pharmacother 110: 609-617, 2019. https://doi.org/10.1016/j.biopha.2018.12.011

PALANIAPPAN L, CARNETHON M, FORTMANN SP: Association between microalbuminuria and the metabolic syndrome: NHANES III. Am J Hypertens 16: 952-958, 2003. https://doi.org/10.1016/S0895-7061(03)01009-4

PAUSOVA Z, PAUS T, SEDOVA L, BERUBE J: Prenatal exposure to nicotine modifies kidney weight and blood pressure in genetically susceptible rats: a case of gene-environment interaction. Kidney Int 64: 829-835, 2003. https://doi.org/10.1046/j.1523-1755.2003.00172.x

PERNG W, KELSEY MM, SAUDER KA, DABELEA D: How does exposure to overnutrition in utero lead to childhood adiposity? Testing the insulin hypersecretion hypothesis in the EPOCH cohort. Diabetologia 64: 2237-2246, 2021. https://doi.org/10.1007/s00125-021-05515-2

REAVEN GM: Banting lecture 1988. Role of insulin resistance in human disease. Diabetes 37: 1595-1607, 1988. https://doi.org/10.2337/diabetes.37.12.1595

RODRIGO S, FAUSTE E, DE LA CUESTA M, RODRIGUEZ L, ALVAREZ-MILLAN JJ, PANADERO MI, OTERO $\mathrm{P}, \mathrm{BOCOS} \mathrm{C}$ : Maternal fructose induces gender-dependent changes in both LXRalpha promoter methylation and cholesterol metabolism in progeny. J Nutr Biochem 61: 163-172, 2018. https://doi.org/10.1016/j.jnutbio.2018.08.011

RODRIGUEZ L, OTERO P, PANADERO MI, RODRIGO S, ALVAREZ-MILLAN JJ, BOCOS C: Maternal fructose intake induces insulin resistance and oxidative stress in male, but not female, offspring. J Nutr Metab 2015: 158091, 2015. https://doi.org/10.1155/2015/158091

RODRIGUEZ L, PANADERO MI, ROGLANS N, OTERO P, ALVAREZ-MILLAN JJ, LAGUNA JC, BOCOS C: Fructose during pregnancy affects maternal and fetal leptin signaling. J Nutr Biochem 24: 1709-1716, 2013. https://doi.org/10.1016/j.jnutbio.2013.02.011

SAKLAYEN MG: The Global Epidemic of the Metabolic Syndrome. Curr Hypertens Rep 20: 12, 2018. https://doi.org/10.1007/s11906-018-0812-Z

SARKAR A, YOO JY, VALERIA OZORIO DUTRA S, MORGAN KH, GROER M: The association between earlylife gut microbiota and long-term health and diseases. J Clin Med 10: 459, 2021. https://doi.org/10.3390/jcm10030459

SEDA O, LISKA F, KRENOVA D, KAZDOVA L, SEDOVA L, ZIMA T, PENG J, PELINKOVA K, TREMBLAY J, HAMET P, KREN V: Dynamic genetic architecture of metabolic syndrome attributes in the rat. Physiol Genomics 21: 243-252, 2005. https://doi.org/10.1152/physiolgenomics.00230.2004 
SEDA O, SEDOVA L, KAZDOVA L, KRENOVA D, KREN V: Metabolic characterization of insulin resistance syndrome feature loci in three brown Norway-derived congenic strains. Folia Biol (Praha) 48: 81-88, 2002.

SEDA O, SEDOVA L, VCELAK J, VANKOVA M, LISKA F, BENDLOVA B: ZBTB16 and metabolic syndrome: a network perspective. Physiol Res 66: S357-S365, 2017. https://doi.org/10.33549/physiolres.933730

SEDA O, TREMBLAY J, GAUDET D, BRUNELLE PL, GURAU A, MERLO E, PILOTE L, ORLOV SN, BOULVA F, PETROVICH M, KOTCHEN TA, COWLEY AW, JR., HAMET P: Systematic, genome-wide, sex-specific linkage of cardiovascular traits in French Canadians. Hypertension 51: 1156-1162, 2008. https://doi.org/10.1161/HYPERTENSIONAHA.107.105247.

SEDOVA L, KAZDOVA L, SEDA O, KRENOVA D, KREN V: Rat inbred PD/cub strain as a model of dyslipidemia and insulin resistance. Folia Biol (Praha) 46: 99-106, 2000.

SEDOVA L, PROCHAZKA J, ZUDOVA D, BENDLOVA B, VCELAK J, SEDLACEK R, SEDA O: Heterozygous Nme7 Mutation Affects Glucose Tolerance in Male Rats. Genes (Basel) 12: 1087, 2021. https://doi.org/10.3390/genes12071087

SEDOVA L, SEDA O, KAZDOVA L, CHYLIKOVA B, HAMET P, TREMBLAY J, KREN V, KRENOVA D: Sucrose feeding during pregnancy and lactation elicits distinct metabolic response in offspring of an inbred genetic model of metabolic syndrome. Am J Physiol Endocrinol Metab 292: E1318-1324, 2007. https://doi.org/10.1152/ajpendo.00526.2006

SEONG HY, CHO HM, KIM M, KIM I: Maternal High-Fructose Intake Induces Multigenerational Activation of the $\begin{array}{lllll}\text { Renin-Angiotensin-Aldosterone } & \text { System. } & \text { Hypertension } & 74: & 518-525,\end{array}$ https://doi.org/10.1161/HYPERTENSIONAHA.119.12941

SHAMANSUROVA Z, TAN P, AHMED B, PEPIN E, SEDA O, LAVOIE JL: Adipose tissue (P)RR regulates insulin sensitivity, fat mass and body weight. Mol Metab 5: 959-969, 2016. https://doi.org/10.1016/j.molmet.2016.08.009

SHARP GC, LAWLOR DA: Paternal impact on the life course development of obesity and type 2 diabetes in the offspring. Diabetologia 62: 1802-1810, 2019. https://doi.org/10.1007/s00125-019-4919-9

SKOLNIKOVA E, SEDOVA L, CHYLIKOVA B, KABELOVA A, LISKA F, SEDA O: Maternal High-Sucrose Diet Affects Phenotype Outcome in Adult Male Offspring: Role of Zbtb16. Front Genet 11: 529421, 2020a. https://doi.org/10.3389/fgene.2020.529421

SKOLNIKOVA E, SEDOVA L, LISKA F, SEDA O: SHR-Zbtb16 minimal congenic strain reveals nutrigenetic interaction between Zbtb16 and high-sucrose diet. Physiol Res 69: 521-527, 2020b. https://doi.org/10.33549/physiolres.934423

SKOLNIKOVA E, SEDOVA L, SEDA O: Grandmother's Diet Matters: Early Life Programming with Sucrose Influences Metabolic and Lipid Parameters in Second Generation of Rats. Nutrients 12: 846, 2020c. https://doi.org/10.3390/nu12030846

SLOBODA DM, LI M, PATEL R, CLAYTON ZE, YAP C, VICKERS MH: Early life exposure to fructose and offspring phenotype: implications for long term metabolic homeostasis. J Obes 2014: 203474, 2014. https://doi.org/10.1155/2014/203474

SOFTIC S, GUPTA MK, WANG G-X, FUJISAKA S, O'NEILL BT, RAO TN, WILLOUGHBY J, HARBISON C, FITZGERALD K, ILKAYEVA O, NEWGARD CB, COHEN DE, KAHN CR: Divergent effects of glucose and fructose on hepatic lipogenesis and insulin signaling. The Journal of Clinical Investigation 127: 4059-4074, 2017. https://doi.org/10.1172/JCI94585

SOUBRY A: POHaD: why we should study future fathers. Environ Epigenet 4: dvy007, 2018. https://doi.org/10.1093/eep/dvy007

TAIN YL, HSU CN, CHAN JY, HUANG LT: Renal Transcriptome Analysis of Programmed Hypertension Induced by Maternal Nutritional Insults. Int J Mol Sci 16: 17826-17837, 2015. https://doi.org/10.3390/ijms160817826

TAIN YL, LEE WC, WU KLH, LEU S, CHAN JYH: maternal high fructose intake increases the vulnerability to postweaning high-fat diet-induced programmed hypertension in male offspring. Nutrients 10: 56, 2018. https://doi.org/10.3390/nu10010056 
TAM WH, MA RCW, OZAKI R, LI AM, CHAN MHM, YUEN LY, LAO TTH, YANG X, HO CS, TUTINO GE, CHAN JCN: In utero exposure to maternal hyperglycemia increases childhood cardiometabolic risk in offspring. Diabetes Care 40: 679-686, 2017. https://doi.org/10.2337/dc16-2397

TAYLOR SR, RAMSAMOOJ S, LIANG RJ, KATTI A, POZOVSKIY R, VASAN N, HWANG SK, NAHIYAAN N, FRANCOEUR NJ, SCHATOFF EM, JOHNSON JL, SHAH MA, DANNENBERG AJ, SEBRA RP, DOW LE, CANTLEY LC, RHEE KY, GONCALVES MD: Dietary fructose improves intestinal cell survival and nutrient absorption. Nature 597: 263-267, 2021. https://doi.org/10.1038/s41586-021-03827-2

TOBAR-BERNAL FA, ZAMUDIO SR, QUEVEDO-CORONA L: The high-fructose intake of dams during pregnancy and lactation exerts sex-specific effects on adult rat offspring metabolism. J Dev Orig Health Dis 12: 411-419, 2021. https://doi.org/10.1017/S2040174420000501

TOOP CR, MUHLHAUSLER BS, O'DEA K, GENTILI S: Impact of perinatal exposure to sucrose or high fructose corn syrup (HFCS-55) on adiposity and hepatic lipid composition in rat offspring. J Physiol 595: 4379-4398, 2017. https://doi.org/10.1113/JP274066

VERMEULEN R, SCHYMANSKI EL, BARABASI AL, MILLER GW: The exposome and health: Where chemistry meets biology. Science 367: 392-396, 2020. https://doi.org/10.1126/science.aay3164

VICKERS MH, CLAYTON ZE, YAP C, SLOBODA DM: Maternal fructose intake during pregnancy and lactation alters placental growth and leads to sex-specific changes in fetal and neonatal endocrine function. Endocrinology 152: 1378-1387, 2011. https://doi.org/10.1210/en.2010-1093

WU C, LI J, BO L, GAO Q, ZHU Z, LI D, LI S, SUN M, MAO C, XU Z: High-sucrose diets in pregnancy alter angiotensin II-mediated pressor response and microvessel tone via the $\mathrm{PKC} / \mathrm{Cav} 1.2$ pathway in rat offspring. Hypertens Res 37: 818-823, 2014. https://doi.org/10.1038/hr.2014.94

WU L, SHI A, ZHU D, BO L, ZHONG Y, WANG J, XU Z, MAO C: High sucrose intake during gestation increases angiotensin II type 1 receptor-mediated vascular contractility associated with epigenetic alterations in aged offspring rats. Peptides 86: 133-144, 2016. https://doi.org/10.1016/j.peptides.2016.11.002

YANAI H, ADACHI H, HAKOSHIMA M, KATSUYAMA H: Molecular Biological and Clinical Understanding of the Pathophysiology and Treatments of Hyperuricemia and Its Association with Metabolic Syndrome, Cardiovascular Diseases and Chronic Kidney Disease. Int J Mol Sci 22: 9221, 2021. https://doi.org/10.3390/ijms22179221

YURUK AA, NERGIZ-UNAL R: Maternal dietary free or bound fructose diversely influence developmental programming of lipogenesis. Lipids Health Dis 16: 226, 2017. https://doi.org/10.1186/s12944-017-0618-Z

ZAK A, BURDA M, VECKA M, ZEMAN M, TVRZICKA E, STANKOVA B: Fatty acid composition indicates two types of metabolic syndrome independent of clinical and laboratory parameters. Physiol Res 63: S375-385, 2014. https://doi.org/10.33549/physiolres.932868

ZHANG P, ZHU D, ZHANG Y, LI L, CHEN X, ZHANG W, SHI R, TAO J, HAN B, XU Z: Synergetic Effects of Prenatal and Postnatal High Sucrose Intake on Glucose Tolerance and Hepatic Insulin Resistance in Rat Offspring. Mol Nutr Food Res 62: 1700771, 2018. https://doi.org/10.1002/mnfr.201700771 